Kasuistik

$\mathrm{D}$ ie schnelle und wirksame Behandlung der akuten Manie bipolarer und schizoaffektiver Patienten ist von entscheidender Bedeutung für den Krankheitsverlauf, das soziale Funktionsniveau und die Behandlungskosten der Patienten (6).

Eine Substanz zur Behandlung der Manie sollte daher folgende Kriterien erfüllen (7): Zuerst sollte sie möglichst wirkungsvoll die manischen, sowie eventuell vorhandene psychotische und depressive Symptome bekämpfen. Dabei sind ein schneller Wirkungseintritt sowie die Wirkungsstärke der Substanz zur Behandlung der akuten Manie entscheidend. Die Substanz sollte die Verbesserung der Symptome stabilisieren und erhalten, sowie akute Rückfalle verhindern können (Continuation). Eine phasenprophylaktische Behandlung schließt sich an, die durch das antimanische Medikament begonnen oder bei bereits bestehender phasenprophylaktischer Medikation durch die antimanische Substanz verbessert werden soll (Maintenance). Dabei sollte das Risiko einer möglicherweise der Manie folgenden Depression minimiert werden. $\mathrm{Zu}$ all diesen funktionellen Anforderungen an eine antimanische Substanz kommen folgende klinisch relevanten Gesichtspunkte dazu: Die Nebenwirkungen sollten möglichst gering sein, auch, um die Compliance der Patienten zu erhöhen. Es empfiehlt sich eine einfache Dosierung und Darreichungsform, in einzelnen Fällen wäre eine parenterale Applikationsform wichtig. Bei der Auswahl einer antimanischen Substanz sollte die Anzahl anfallender Blut- und Blutspiegelkontrollen beachtet werden, ebenso wie etwaige Wechselwirkungen mit anderen Medikamenten. Ein weiterer wichtiger Punkt ist die Verträglichkeit der Substanz im Hinblick auf somatische Erkrankungen.

Der folgende Fallbericht beschreibt eine Patientin, die in einer akuten manischen Episode prospektiv monotherapeutisch mit Risperidon behandelt wurde. Dabei bestanden bei der Auswahl der antimanischen Substanz aufgrund zahlreicher somatischer Vorerkrankungen

\title{
Risperidon: akute Manie bei multiplen somatischen Vorerkrankungen
}

\author{
Anna Forsthoff', Frank-Gerald Pajonk², Heinz Grunze ${ }^{1}$ \\ ${ }^{1}$ Psychiatrische Klinik der Universität München \\ 2Nervenklinik - Psychiatrie und Psychotherapie, Universitätskliniken des Saarlandes
}

Atypische Neuroleptika nehmen in der Behandlung der akuten Manie bipolarer und schizoaffektiver Störungen eine zunehmend wichtige Stellung ein. Der folgende Fallbericht beschreibt eine Patientin, die in einer akuten manischen Episode prospektiv monotherapeutisch mit Risperidon behandelt wurde. Dabei bestanden bei der Auswahl der antimanischen Substanz aufgrund zahlreicher somatischer Vorerkrankungen der Patientin besondere Schwierigkeiten und Anforderungen. Der Einsatz von Risperidon führte erfolgreich und gut verträglich zur raschen Besserung der maniformen Symptomatik.

der Patientin besondere Schwierigkeiten und Anforderungen.

\section{- Kasuistik}

Frau I. ist eine 54-jährige verheiratete Hausfrau, die sich im Dezember 2001 mit einem maniformen Syndrom in unserer Ambulanz vorstellte und auf die Akutstation aufgenommen wurde, wo sie freiwillig verblieb.

Bei Frau I. war seit 1986 eine rezidivierende depressive Störung bekannt. Im Rahmen dieser Erkrankung kam es zu zahlreichen stationär psychiatrischen Aufenthalten. Laut Aussagen des Ehemannes zeigten sich jedoch in den letzten Jahren Episoden gehobener Stimmung mit unvernünftigen Einkäufen und desorganisiertem Verhalten. Eine solche Phase führte im Jahr $2000 \mathrm{zu}$ einem stationären Aufenthalt. Frau I. wurde damals mit Haloperidol 5 mg/d erfolgreich behandelt. Eine phasenprophylaktische Behandlung wurde aufgrund zahlreicher internistischer Erkrankungen (s.u.) nicht empfohlen. Nach einem Zeitraum von etwa einem Jahr kam es nach Angaben des Ehemannes wieder zu vermehrten Geldausgaben, Frau I. war zunehmend stimmungsgehoben, umtriebig, desorganisiert und gereizt. Nach einem körperlichen Angriff auf den Ehemann, brachte dieser die Patientin in die Klinik.

Die somatische Vorgeschichte der Patientin beschreibt eine hypertensive Herzerkrankung mit relativer Mitralinsuffizienz. Zusätzlich ist ein systemischer Lupus Erythematodes bekannt. Aufgrund einer Struma nodosa mit autonomem Adenom wurde 1992 eine Strumateilresektion durchgeführt. Als notwendige Dauermedikation erhielt Frau I. L-Thyroxin $100 \mu \mathrm{g} / \mathrm{d}$, Verapamil $120 \mathrm{mg} / \mathrm{d}$, Furosemid $30 \mathrm{mg} / \mathrm{d}$, Spironolacton 200 $\mathrm{mg} / \mathrm{d}$ und Phenprocoumon entsprechend des Quick-Wertes.

Bei Aufnahme zeigte sich Frau I. wach, bewusstseinsklar und zeitlich unscharf orientiert. Ihr Verhalten wirkte stark desorganisiert. Sie war übertrieben geschminkt, bizarr geschmückt und auffällig gekleidet. Es bestanden deutliche Aufmerksamkeits- und Konzentrationsstörungen. Im formalen Gedankengang war sie beschleunigt, ideenflüchtig und vorbeiredend, zum Teil auch denkzerfahren. Inhaltlich ergaben sich Hinweise auf psychotisches Erleben in Form von Beziehungs- und Beeinträchtigungswahn, eine klare Exploration war jedoch aufgrund der formalen Denkstörung nicht möglich. Ich-Störungen und Halluzinationen wurden von der Patientin verneint 


\section{Abb. 1 Verlauf der maniformen Symptomatik}

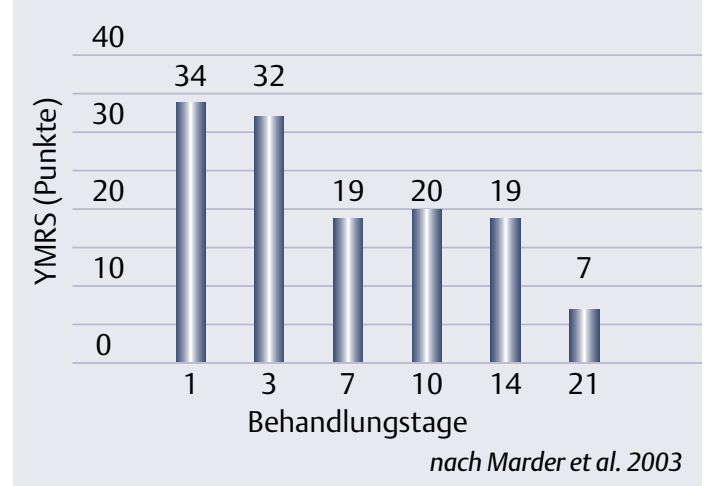

Abb.1: Reduktion der maniformen Symptomatik gemessen mit der Young Mania Rating Scale (YMRS)

\section{Abb. 2 Krankheitsschwere und Risperidon-Dosis}

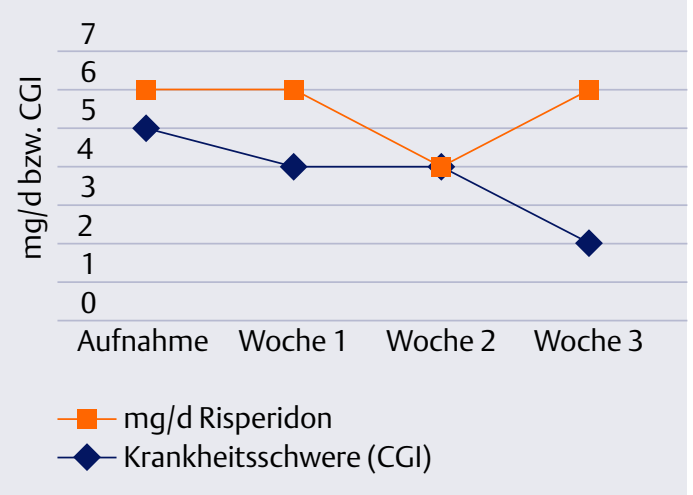

Abb. 2: Entwicklung des Clinical Global Impression Scores (CGI) in Abhängigkeit von der verabreichten Risperidon Tagesdosis (die Dosis wurde ab der 3. Woche langsam auf $4 \mathrm{mg} / \mathrm{d}$ reduziert und die Medikation so fortgeführt)

ebenso Phobien oder Zwänge. Affektiv war sie euphorisch und lachte inadäquat über den ihr völlig unverständlichen Aufwand um ihre Person, teils war sie dysphor, leicht reizbar mit verbal aggressiven Ausbrüchen. Der Antrieb und die Psychomotorik waren gesteigert. Frau I. klagte über Schlaflosigkeit. Suizidalität verneinte die Patientin. Obwohl Frau I. nur bedingt Krankheitseinsicht zeigte, war sie dennoch behandlungsbereit.

Nach Ausschluss organischer Ursachen für die psychische Störung wurde eine bipolar affektive Störung, gegenwärtig manische Episode, mit psychotischen Symptomen diagnostiziert (ICD-10: F 31.20; DSM-IV: 296.44).
Der Schweregrad der manischen Symptomatik und der Verlauf der Behandlung wurden mittels der Young Mania Rating Scale (YMRS) sowie der Clinical Global Impression Scale (CGIBP) verfolgt (Abb. 1 und 2).

Eine medikamentöse Behandlung wurde einschleichend mit $4 \mathrm{mg}$ Risperidon pro Tag begonnen und rasch auf $6 \mathrm{mg} / \mathrm{d}$ erhöht. Als Zusatzmedikation erhielt die Patientin Lorazepam $3 \mathrm{mg} / \mathrm{d}$. Zu Beginn der Untersuchung erreichte Frau I. im YMRS einen Wert von 34 Punkten (Tag 1 , siehe Abb. 1), welcher am dritten Tag auf 32 absank. Die Risperidon-Dosis mit $6 \mathrm{mg} / \mathrm{d}$ wurde am 11. Tag auf $4 \mathrm{mg} / \mathrm{d}$ reduziert, da die Patientin über eine Akathisie klagte, die nach der Dosisreduktion verschwand. Eine Besserung der manischen Symptomatik zeigte sich nach einer Woche, der YMRS zeigte an den Tagen sieben und zehn einen Wert von 19 bzw. 20. Da die maniforme Symptomatik nach der Dosisreduktion zwar gebessert war, aber dennoch weiterhin bestand (siehe Abbildung 1), wurde Risperidon am 16. Tag wieder auf $6 \mathrm{mg} / \mathrm{d}$ erhöht, bei weiterhin guter Verträglichkeit und ohne erneutes Auftreten der Akathisie. Daraufhin verbesserte sich das maniforme Bild, Frau I. wurde an Tag $21 \mathrm{im}$ YMRS mit sieben Punkten eingestuft. Die Risperidon-Dosis wurde dann langsam reduziert und mit $4 \mathrm{mg} / \mathrm{d}$ fortgeführt.

Insgesamt stellte sich eine deutliche psychopathologische Besserung nach einer Woche ein, Frau I. wurde zunehmend formal geordneter, die inhaltlichen Beziehungs- und Beeinträchtigungsideen wurden von ihr reflektiert und infrage gestellt. Die Stimmung war noch gehoben, leicht gereizt und unterschwellig sarkastisch und provokant. Die zweite Woche zeigte weitgehend dasselbe psychopathologische Bild, weshalb eine Erhöhung der Risperidon-Dosis erfolgte. Nach etwa zwei Wochen verbesserte sich das maniforme Bild zunehmend, Frau I. konnte wieder durchschlafen, im Affekt war sie ausgeglichen und kaum noch gereizt. Frau I. war weiterhin auffällig gekleidet, ihr Verhalten wirkte nun aber eher theatralisch und histrionisch anmutend. Frau I. zeigte sich sehr kooperativ, krankheitseinsichtig und hatte realistische Zukunftspläne.

Nach vier Wochen konnten wir Frau I. in die ambulante Behandlung nach Hause entlassen. Eine Weiterführung der Medikation mit Risperidon $4 \mathrm{mg} / \mathrm{d}$, wurde im Sinne einer Phasenprophylaxe empfohlen.

\section{Diskussion}

Bisherige Behandlungsstrategien akuter manischer Episoden bei bipolaren oder schizoaffektiven Patienten empfehlen die Gabe von Mood-Stabilizern wie Lithium, Valproinsäure oder Carbamazepin in allen Phasen der Behandlung. Dazu werden bei nicht ausreichendem Ansprechen der Stimmungsstabilisierer Neuroleptika sowie Benzodiazepine kombiniert (11). Die Wirksamkeit der einzelnen Mood-Stabilizer sowie deren Kombination, ist in vielen Studien gut dokumentiert (7). Untersuchungen von Neuroleptika in Kombination mit Mood-Stabilizern in der Behandlung der akuten Manie bipolarer und schizoaffektiver Störungen sind relativ zahlreich, und zeigen eine zunehmend bedeutende Stellung neuerer Neuroleptika in der Maniebehandlung (4). Einige Untersuchungen sind beschrieben, die die antimanische Wirksamkeit der atypischen Neuroleptika in einer Monotherapie untersuchen $(8,12,13,16)$.

In der Literatur finden sich zahlreiche Hinweise, dass Risperidon die o.a. Kriterien an ein Psychopharmakon zur Behandlung akuter manischer Symptome weitgehend erfüllt. So konnte die Wirksamkeit von Risperidon auf sowohl depressive wie auch maniforme Symptome bei schizophrenen Patienten gezeigt werden (10). Auch in der Behandlung der akuten Manie spielt Risperidon eine zunehmende Rolle. Viele Arbeiten, in denen die Substanz als Monotherapie oder als Zusatzmedikation zu Stimmungsstabilisierern untersucht wurde, zeigen eine signifikante Wirksamkeit, sowohl in der Akut- wie auch der Erhaltungstherapie $(2,3,12,14,16)$.

Die mit diesem Fall beschriebene Patientin wurde in einer akuten manischen Episode erfolgreich monotherapeutisch mit Risperidon in Do- 
sierungen zwischen 4 und $6 \mathrm{mg} / \mathrm{d}$ behandelt. Bei der Wahl der antimanischen Substanz bestanden in diesem Fall besondere Schwierigkeiten und Anforderungen.

Aufgrund der bei der Patientin bestehenden somatischen Vorerkrankungen kam eine Behandlung der Manie mit einem klassischen Stimmungsstabilisierer nicht in Frage. Lithium konnte aufgrund des bestehenden systemischen Lupus erythematodes (SLE), sowie potenziell gefährlichen Wechselwirkungen mit dem Aldosteronantagonisten sowie dem Schleifendiuretikum, und einer damit verbundenen Potenzierung der Nephrotoxizität, nicht gegeben werden (9). Valproat und Carbamazepin bergen die Gefahr allergischer Hautveränderungen (5), Valproat ist beim SLE kontraindiziert. Aufgrund der Notwendigkeit einer Marcumarisierung der Patientin und der möglichen Hepatotoxizität von Valproat und Carbamazepin (1) waren diese beiden Substanzen nicht geeignet.

Risperidon stellt eine Substanz dar, die durch zuverlässige Wirksamkeit, gute Verträglichkeit sowie geringe Wechselwirkungen mit anderen Präparaten gekennzeichnet ist (15). Diese Eigenschaften haben sich mittlerweile in zahlreichen offenen und doppel-blinden Studien an bipolaren Patienten bestätigt. Auch die Rate an extrapyramidalmotorischen Nebenwirkungen ist in adäquater Dosierung bis zu $6 \mathrm{mg} / \mathrm{d}$ im Vergleich zu anderen Neuroleptika relativ gering (13).

Im vorliegenden Fall lässt sich zusammenfassend feststellen, dass die Behandlung mit Risperidon erfolgreich war. Die antimanische Wirkung war effektiv und in Hinblick auf die Begleiterkrankungen der Patientin war die Substanz als sicher einzustufen. Ob Risperidon in niedriger Dosierung eine phasenprophylaktische Wirkung bei dieser Patientin zeigt, bleibt offen; um die beschriebenen Vorteile dieser Substanz zu bestätigen, sind weitere Untersuchungen notwendig.

\section{Literatur}

1. Dreifuss FE, Santilli N, Langer H. Valproic acid hepatic facilities: a retrospective review. Neurology 1987; 37: 379-385

2. Ghaemi SN, Sachs GS, Baldassano CF, Truman Cl. Acute Treatment of Bipolar Disorder with Adjunctive Risperidone in Outpatients. Can J Psychiatry 1997; 42: 196-199

3. Ghaemi SN, Sachs GS. Long-term risperidone treatment in bipolar disorder: 6month follow up. Int Clin Psychopharmacol 1997; 12: 333-338

4. Ghaemi SN. New treatments for bipolar disorder: the role of atypical neuroleptic agents. J Clin Psychiatry 2000; 61 Suppl 14: 33-42

5. Grunze H, Walden J. Valproat bei manisch-depressiven Erkrankungen. Stuttgart, Thieme 2000

6. Keck PE, McElroy SL, Benett JA. Health economic implications of the onset of action of antimanic agents. J Clin Psychiatry 1996; 57 (Suppl 13):13-28

7. Licht RW. Drug treatment of Mania: a critical review. Acta Psychiatry Scand 1998; 97: 387-397

8. Licht RW, Bysted M, Christensen H. Fixed-dosed risperidone in mania: an open experimental trial. Int Clin Psychopharmacol 2001; 16: 103-110

9. Müller-Oerlinghausen B, Greil W, Berghöfer A. Die Lithiumtherapie - Nutzen, Risiken, Alternativen. Springer 1997

10. Peuskens J, Van Baelen B, De Smedt C, Lemmens P. Effects of risperidone on affective symptoms in patients with schizophrenia. Int Clin Psychopharmacol 2000; 15:343-349

11. Sachs GS, Printz D], Kahn DA, Carpenter D, Docherty JP. The Expert Consensus Guidelines Series: Medication Treatment of Bipolar Disorder 2000. Postgrad Med 2000 Apr; Spec No: 1-104

12. Segal J, Berk M, Brook S. Risperidone compared with both lithium and haloperidol in mania. Clin Neuropharmacol 1998; 21: $176-180$

13. Simpson GM, Lindenmayer J-P. Extrapyramidal Symptoms in patients treated with risperidone. J Clin Psychopharmacol 1997; 17: 194-201

14. Tohen M, Zarate CA, Centorrino $\mathrm{F}$ et al. Risperidone in the treatment of mania. J Clin Psychiatry 1996; 57: 249-253

15. deVane CL, Nemeroff CB. An Evaluation of risperidone drug interactions. J Clin Psychopharmacol 2001; 21: 408-416

16. Vieta E et al. Risperidone Safety and Efficacy in the treatment of bipolar and schizoaffective disorders: results from a 6 months, multicenter, open study. J Clin Psychiatry $2001 ; 62: 818-825$

\section{Korrespondenzadresse:}

Anna Forsthoff

Psychiatrische Klinik der Universität München

Nussbaumstraße 7

80336 München

\section{Aktuell und praxisnah}

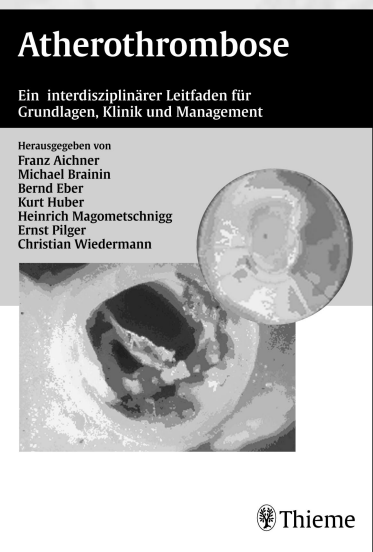

Atherothrombose

Ein interdisziplinärer Leitfaden für Grundlagen, Klinik und Management

Aichner u.a.

Praxisnah:

- Klare therapeutische Handlungsrichtlinien

- Interdisziplinäre Strategien zur Vermeidung und Behandlung der Atherothrombose

- Aktuelle Erkenntnisse aus Sicht der Kardiologie, Neurologie und Gefäßchirurgie

2002. 192 S., 42 Abb., 44 Tab. ISBN $3131298618 €$ 39,95

$0711 / 8931-333$

$0711 / 8931-133$

@ kundenservice@thieme.de www ww.thieme.de 